\title{
PRELIMINARY STUDY ON LOCK-IN THERMOGRAPHY USING AN INVERSE DYNAMIC SYSTEM DESIGN
}

\author{
Hee-Chan Kang ${ }^{1+}$, Hwan Hur ${ }^{1}$, Seon-Jin Kim², Geon-Hee Kim¹, Kye-Sung Lee ${ }^{1 *}$ \\ ${ }^{1}$ Korea Basic Science Institute, Gwahak-ro 168-148, Yuseong-gu, Daejeon, Korea 34133 \\ ${ }^{2}$ ChungNam National University, Daehak-ro 99, Yuseong-gu, Daejeon, Korea 34134 \\ †Presenting Author: hckang90@kbsi.re.kr \\ *Corresponding Author: kslee24@kbsi.re.kr
}

\begin{abstract}
We adopted an inverse dynamical system(IDS) into the Lock-in Thermography(LIT) to increase the sensitivity of the semiconductor inspection system. IDS was designed to obtain the maximum signal to noise ratio(SNR) of LIT image by applying an optimized signal to the semiconductor chip to be inspected using a programmable voltage generator. In addition, we introduce some user friendly features such as monitoring the state of the system and the flexible control of Lock-in frequency and source voltage.
\end{abstract}

KEYWORDS: Infrared, Semiconductor Inspection Equipment, Lock-in Thermography 